Preprint

UCRL-JC-134523 Rev. 1

\title{
A Strength and Damage Model for Rock Under Dynamic Loading
}

O. Yu. Vorobiev, T.H. Antoun, I.N. Lomov and L.A. Glenn

This article was submitted to

American Society of Mechanical Engineers Conference Seattle, WA

July 23-27, 2000

U.S. Department of Energy

\section{December 1, 1999}

Lawrence

Livermore

National

Laboratory 


\section{DISCLAIMER}

This document was prepared as an account of work sponsored by an agency of the United States Government. Neither the United States Government nor the University of California nor any of their employees, makes any warranty, express or implied, or assumes any legal liability or responsibility for the accuracy, completeness, or usefulness of any information, apparatus, product, or process disclosed, or represents that its use would not infringe privately owned rights. Reference herein to any specific commercial product, process, or service by trade name, trademark, manufacturer, or otherwise, does not necessarily constitute or imply its endorsement, recommendation, or favoring by the United States Government or the University of California. The views and opinions of authors expressed herein do not necessarily state or reflect those of the United States Government or the University of California, and shall not be used for advertising or product endorsement purposes.

This is a preprint of a paper intended for publication in a journal or proceedings. Since changes may be made before publication, this preprint is made available with the understanding that it will not be cited or reproduced without the permission of the author.

This report has been reproduced directly from the best available copy.

Available to DOE and DOE contractors from the

Office of Scientific and Technical Information

P.O. Box 62, Oak Ridge, TN 37831

Prices available from (423) 576-8401

http:/ / apollo.osti.gov/bridge/

Available to the public from the National Technical Information Service

U.S. Department of Commerce 5285 Port Royal Rd., Springfield, VA 22161 http://www.ntis.gov/

OR

Lawrence Livermore National Laboratory Technical Information Department's Digital Library http://www.llnl.gov/tid/Library.html 


\title{
A STRENGTH AND DAMAGE MODEL FOR ROCK UNDER DYNAMIC LOADING
}

\author{
Oleg Yu. Vorobiev, Tarabay H. Antoun, Ilya N. Lomov, Lewis A. Glenn \\ Lawrence Livermore National Laboratory, Geophysics and Global Security Division, Livermore, CA 94550
}

\begin{abstract}
A thermodynamically consistent strength and failure model for granite under dynamic loading has been developed and evaluated. The model agrees with static strength measurements and describes the effects of pressure hardening, bulking, shear-enhanced compaction, porous dilation, tensile failure, and failure under compression due to distortional deformations. This paper briefly describes the model and the sensitivity of the simulated response to variations in the model parameters and in the inelastic deformation processes used in different simulations. Numerical simulations of an underground explosion in granite are used in the sensitivity study.
\end{abstract}

\section{INTRODUCTION}

Modeling the dynamic response of rock materials is a challenging area of research. Since most strength measurements in rock materials are performed for intact samples under static conditions, the models based on these data should account for possible scale and rate effects when being applied to simulation of the dynamic response of large-scale rock masses. Unlike intact rock samples, rock masses may contain discontinuities that may reduce the strength.

We assume that the material is isotropic and apply the mathematical structure of plasticity theory to capture the basic features of the mechanical response of geological materials. We use experimental data obtained under static conditions to calibrate the model for small intact samples and then fit rate- and scale- dependent model parameters to describe the dynamic measurements in spherical shock waves for large scale motion of rock masses in underground explosions.

\section{CONSTITUTIVE EQUATIONS}

To model the dynamic response of material to shock wave loading, the system of equations representing the mass, momentum and energy conservation laws is supplemented by the following equation for the unimodular tensor of elastic distortional deformation $\mathbf{B}$ [1].

$$
\dot{\mathbf{B}}=\mathbf{L B}+\mathbf{B} \mathbf{L}^{\mathrm{T}}-\frac{2}{3}(\mathbf{D} \bullet \mathbf{I}) \mathbf{B}-\Gamma_{p}\left[\mathbf{B}-\frac{3 \mathbf{I}}{\mathbf{B}^{-1} \bullet \mathbf{I}}\right]
$$

Using $\mathbf{B}$, the deviatoric stress $\mathbf{T}^{\prime}$ can be expressed as $\mathbf{T}^{\prime}=G \frac{\rho(1-\Phi)}{\rho_{0}}\left(\mathbf{B}-\frac{1}{3}(\mathrm{~B} \bullet \mathrm{I})\right)$, where $G$ is the shear modulus, $\boldsymbol{\rho}_{0}$ and $\boldsymbol{\rho}$ are the initial and the current density and $\Phi$ is the reference porosity. In Eq.(1), $\Gamma_{p}$ specifies the plastic response of the material and is taken to be a function of the von Mises effective stress $\sigma_{e}$ and the yield strength $Y[2]$ : 


$$
\Gamma_{p}=\Gamma_{p 0}\left(\frac{3 G}{\sigma_{e}}\right)\left(\frac{\left\langle\sigma_{e}-Y\right\rangle}{Y_{0}}\right)^{2}
$$

The conservation laws are integrated numerically using the second order Godunov scheme. Eq. (1) is integrated using the velocity gradient tensor $\mathbf{L}$ and it's symmetric part, $\mathbf{D}$, approximated by solving the Riemann problem. More details about the numerical algorithm can be found in [3].

\section{STRENGTH OF MATERIAL}

The physical phenomena that influence the yield strength $\mathbf{Y}$ are taken into account using a multiplicative form with $\mathbf{Y}$ given by:

$$
Y=Y_{0}(\pi, p) F_{1}(\xi, p) F_{2}(p) F_{3}(\Omega, p) F_{4}(\beta, p) F_{5}(\rho, \varepsilon) \frac{1-\phi}{1-\Phi} .
$$

The reference (i.e., virgin) strength of the rock, $Y_{0}(\pi, p)$, is a decreasing function of crack density, $\pi$, which is assumed to be zero for intact rock samples. We assume that at high pressures when the mobility of the rock blocks in jointed rock masses is low, the strength is approaching the value for a single block which is equivalent to the strength of small intact rock samples measured in laboratory conditions.

$$
Y_{0}(\pi, p)=Y_{00}\left[1-\left[1-\exp \left(-\frac{A \pi}{\pi_{0}}\right)\right] \exp \left(-\frac{p}{p_{0}}\right)\right]
$$

$F_{1}$ is specified in terms of hardening parameters $\xi$ by the form $F_{1}=1+\left(k_{1}-1\right) \xi$, where the value of $k_{1}$ gives the maximum strength hardening when $\xi=1$. The hardening parameter $\xi$ is determined by an evolution equation of the form

$$
\dot{\xi}=k_{2}\left[\frac{\dot{\varepsilon_{p}}}{F_{2}(p)}\right](1-\xi)
$$

The pressure hardening function $F_{2}$ is known from the experiments with small samples [4] . Besides eq.(3) it is also used in eq.(5) to avoid the pressure dependence of the slope of stress-strain curve at the beginning of plastic flow.

The damage function $F_{3}$ specified by (6) makes material weak at low pressures $p \leq p_{0}$ once it is damaged.

$$
F_{3}=1+[f(\Omega)-1] \exp \left[-\frac{\langle p\rangle}{p_{0}}\right], f(\Omega)=1-k_{5} \exp \left[-\frac{k_{6}(1-\Omega)^{2}}{\Omega}\right] .
$$

The damage parameter, $\Omega$, used in the function $\left(F_{3}\right)$ is evaluated using the relation 


$$
\dot{\Omega}=\frac{\left\langle T_{\max }-T_{t h}\right\rangle}{\tau_{d a m} Y_{0}} \text { if } \xi=1
$$

where $T_{\max }$ is the most compressive principal stress, $T_{t h}$ is the threshold stress for damage growth, and $\tau_{d a m}$ is a characteristic time for damage. The damage begins to accumulate when the hardening parameter $\xi$ is equal to unity. This happens when von Mises stress reaches the failure surface (see Figure 1). According to eq.(5) more plastic strain is required to reach failure surface at high pressures . This is also observed in experiments [9] .

$F_{4}$ is a function of the Lode angle described in [1] and $F_{5}$ given by (8) models the effect of melting at high pressures, where $\varepsilon_{m}(\rho)$ is the specific melting energy.

$$
F_{5}=1-\operatorname{Min}\left[1, \frac{\varepsilon}{\varepsilon_{m}(\rho)}\right]
$$

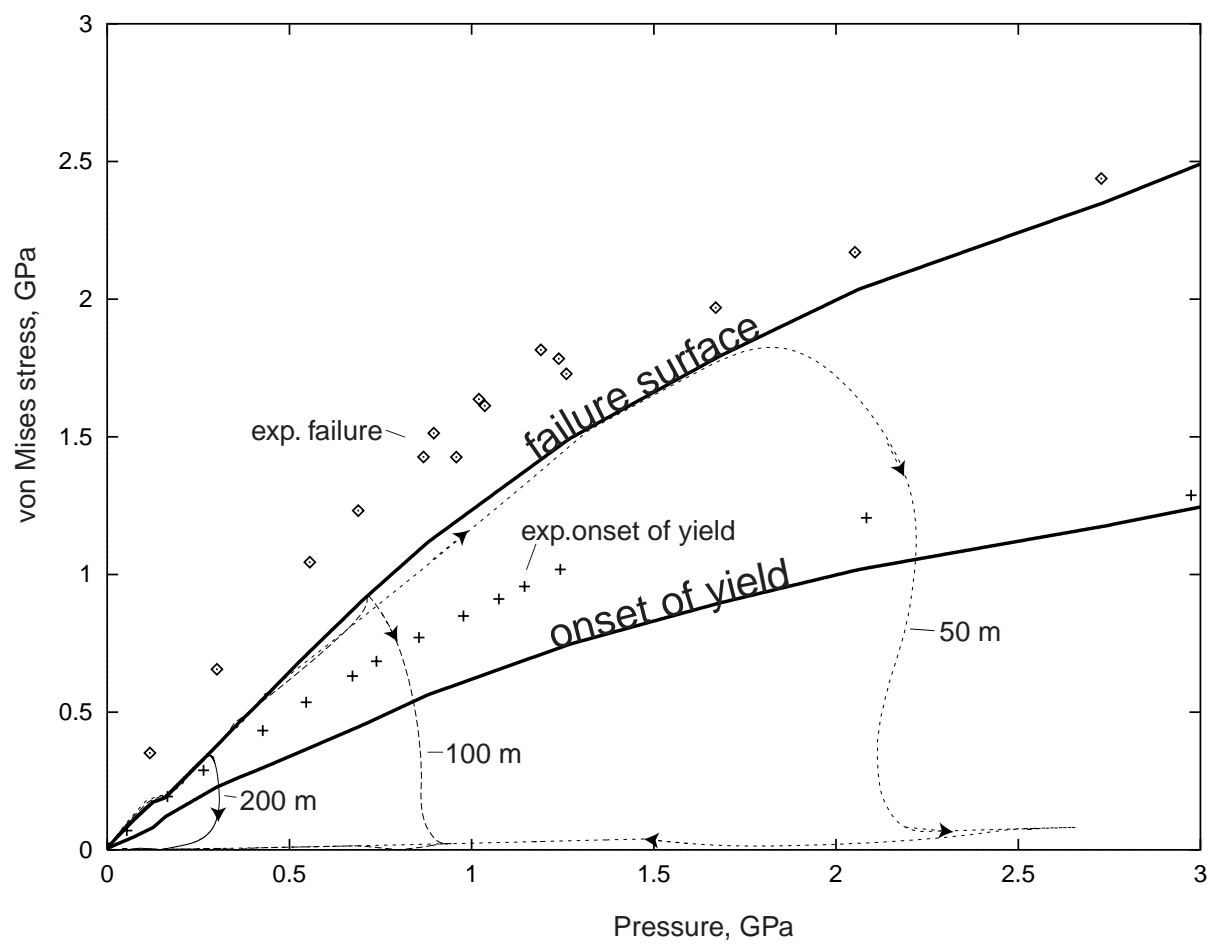

FIGURE 1. Yield and failure surface for granodiorite used in calculations (solid curves) together with the data of static tests [4] (points). 
The total gas porosity is separated into two parts

$$
\phi_{g}=\phi_{g 1}+\phi_{g 2}
$$

The part $\phi_{g 1}$ describes the initial porosity as well as porosity accumulated in tension. The evolution equation for this part is given by (10)

$$
\dot{\phi}_{g 1}=\left\{\begin{array}{ccc}
\Gamma_{d}\left[1+\left(\frac{\phi_{d}^{*}}{\phi_{s}}\right)^{m}\right]\left[\frac{\left\langle\phi_{d}^{*}-\phi_{g}\right\rangle}{\phi_{s}}\right]^{n} & \text { for } & p<-p_{d} \\
0 & \text { for } & -p_{d}<p \leq 0 \\
-\left(1-\phi_{g}\right)\langle-D \bullet I\rangle H\left(\phi_{g 1}-\phi_{g 1 \text { min }}\right) & \text { for } & 0<p \leq p_{c} \\
-\Gamma_{c}\left\langle\phi_{g 1}-\phi_{c}^{*}\right\rangle & \text { for } & p>p_{c}
\end{array}\right.
$$

In eq.(10) $\phi_{g 1 \text { min }}$ is the minimum value attained by $\phi_{g 1}$ during the entire process. The value of $\phi_{d}^{*}$ is found by inverting a linearized equation of state (11) which would give the pressure $p=-p_{d}$ at given volume $J$ and specific energy $\varepsilon$.

$$
\phi_{d}^{*}=1-\frac{\left(1-\Phi_{g}\right) \rho C^{2} / J+p_{d}}{\rho C^{2}-\Gamma \varepsilon \rho}
$$

In eq.(11) $C$ is the sound speed and $\Gamma$ is Gruneisen coefficient.

The value $\phi_{c}^{*}$ is a function of volume and porosity (12) that is used to characterize the compaction curve.

$$
\phi_{c}^{*}=\left\{\begin{array}{ccc}
\Phi_{g 1} & \text { for } & 1 / J<1 / J_{c} \\
\Phi_{g 1}-c_{1}\left(1-\Phi_{g}\right)\left[\frac{1}{J}-\frac{1}{J_{c}}\right] F_{8} & \text { for } & 1 / J_{c}<1 / J<c_{2} / J_{c} \\
\bar{\phi} \exp \left[-\frac{c_{1}\left(1-\Phi_{g}\right)\left\langle\frac{1}{J}-\frac{c_{2}}{J_{c}}\right\rangle F_{8}}{\bar{\phi}}\right] & \text { for } & c_{2} / J_{c}<1 / J
\end{array}\right.
$$

Function $\quad F_{8}$ in eq.(12) models effect of shear enhanced compaction and is expressed as

$$
F_{8}=\left[1+c_{3}\left(\frac{1}{c_{1}}-1\right)\right]-c_{3}\left(\frac{1}{c_{1}}-1\right)\left[\operatorname{Max}\left\{0,1-\left(\sigma_{e} / Y\right)^{2}\right\}\right]^{c_{4}}
$$

Other parameters in eq.(12) are given by 


$$
\begin{aligned}
& \bar{\phi}_{c}=\Phi_{g 1}-c_{1}\left(1-\Phi_{g}\right)\left[\frac{c_{2}}{J_{c}}-\frac{1}{J_{c}}\right] F_{8}, \frac{1}{J_{c}}=\frac{p_{c}}{\left(1-\Phi_{g}\right) \rho C^{2}}+\left(\frac{1-\Phi_{g 1}-\phi_{g 2}}{1-\Phi_{g}}\right)\left\langle 1-\frac{\Gamma \varepsilon}{C^{2}}\right\rangle, 0 \leq c_{1} \leq 1, \quad 0 \leq c_{3} \leq 1, \\
& 1 \leq c_{2} \leq 1+\frac{\Phi_{g 1} J_{c}}{c_{1}\left(1-\Phi_{g 1}\right) F_{8}}, \quad c_{4} \geq 0
\end{aligned}
$$

The part $\phi_{g 2}$ models the porosity due to bulking. The evolution of bulking porosity is given by eq.(15).

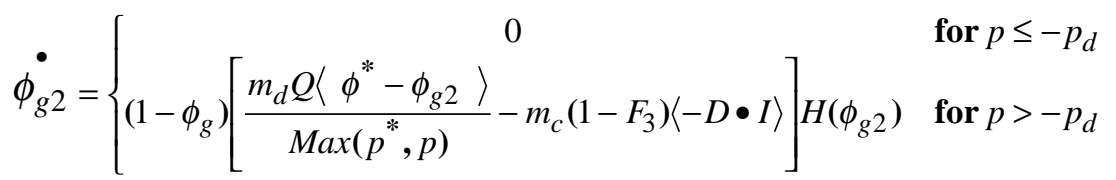

The value of $\phi^{*}$ specifies the maximum bulking porosity that can be achieved, the function $m_{d}$ determines the rate of bulking and $m_{c}$ is used to control the rate of recompaction of the bulking porosity which takes place when material is damaged and $F_{3} \rightarrow 0$. The rate of dissipation $\mathrm{Q}$ due to plastic deformations in eq.(15) is given as

$$
Q=\frac{\rho(1-\Phi)}{2 \rho_{0}} \Gamma_{p} G\left(B^{\prime} \bullet B^{\prime}\right)
$$

Figure 2 shows how well it is possible to fit this model to laboratory bulking data.

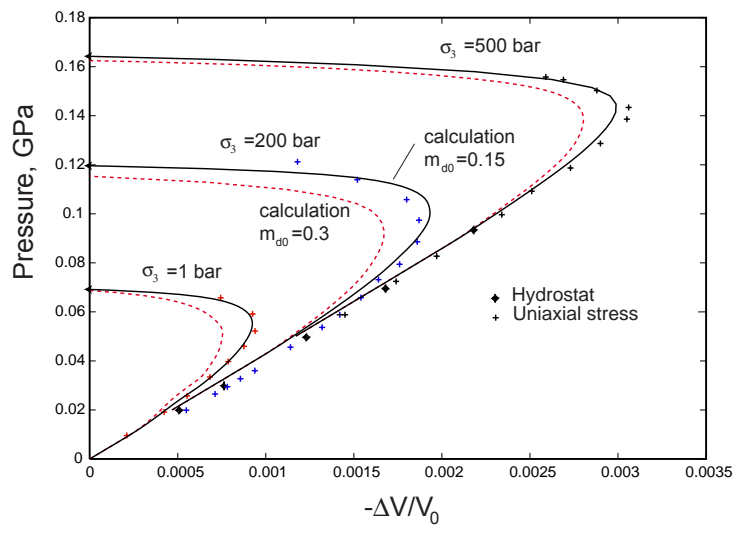

FIGURE 2. Volume strain as a function of pressure for granodiorite during uniaxial stress loading at several confining pressures. The points are experimental data [4], the solid and dashed lines are calculations with different values for $m_{d 0}$. 
To study the effect of the model parameters on the material response in a large scale motion we have simulated a spherical explosion in granite with different yield strength functions. The divergent flow of spherical shock loading leads to a wide variety of stress states in contrast to plane waves, where the locus of all states is represented by a straight line in yield-pressure space. The source was modeled using ideal gas with granite density. We used a Mie-Grüneisen EOS for the granite. A more general tabular EOS was subsequently employed and produced similar results. We found that the first four functions in eq.(3) determine the response of the material the most. The strain and pressure hardening functions are taken to fit the static excperiments [4]. We assume that the main difference in strength is due to the existence of cracks in joint rock masses modeled by $Y_{0}(\pi, p)$ function. The rate dependence of damage modeled by function $F_{3}$. It has been shown in previous research $[5,9]$ that yield strength degradation is required to obtain a deep and wide rebound signal. Since the typical time of events for large scale motion is of an order of $1 \mathrm{~s}$, choosing $\tau_{d a m}$ a smaller value will lead to required damage.

Results from the spherical explosion simulations are shown in Figures 3 and 4 . Figure 3 compares simulated and measured particle velocity and particle displacement histories at two different ranges away from the source. Figure 4 compares simulated and measured peak velocity and displacement attenuation as a function of slant range.

The velocity waveforms are characterized by a positive phase representing the outward motion of the rock, followed by a rebound phase during which the material contracts and displaces radially inward toward the explosive source. Analysis of the simulation results made it possible to associate processes in the constitutive model with measured waveform features. For instance, the peak particle velocity attenuation as a function of scaled slant range, shown in Figure 3(a), is strongly influenced by porous compaction (in addition to its characteristic dependence on the divergent flow field). This attenuation is further complicated by the yielding and damage processes that determine the residual strength of the material behind the shock front. A stronger material allows more of the release waves emanating from the explosive source to catch up with the main shock front and cause it to attenuate at a faster rate, thereby diminishing the peak velocity amplitude.

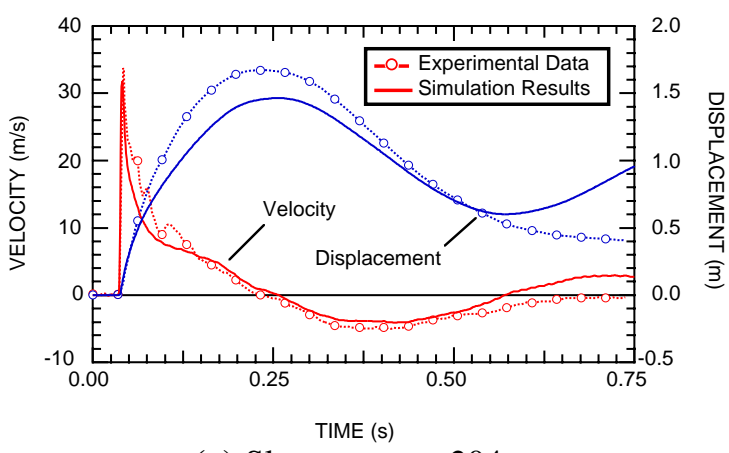

(a) Slant range $=204 \mathrm{~m}$

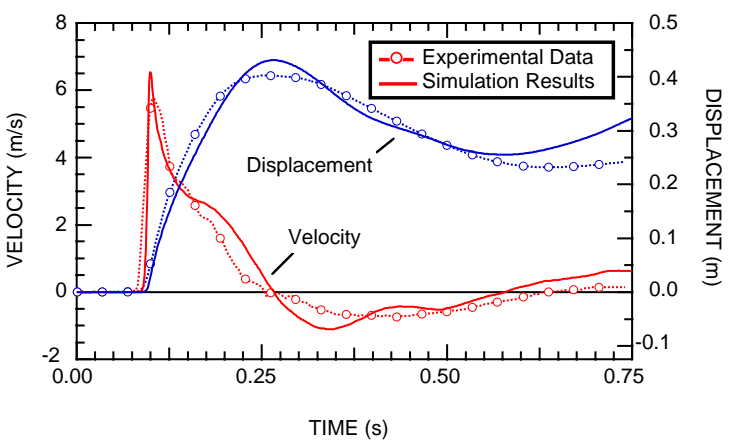

(b) Slant range $=470 \mathrm{~m}$

FIGURE 3. Comparison of simulated and measured radial velocity and displacement histories from an underground explosion in granite.

The width of the positive phase of the velocity waveform is strongly dependent on bulking. The increased volume associated with bulking causes the pressure in the material to be higher than it would be 
if bulking was suppressed. The work done by this higher pressure causes an increase in the outward displacement of the rock. This effect is manifested as a widening of the positive phase of the simulated velocity waveforms. It is also manifested as an increase in the peak displacement observed at various ranges away from the explosive source. The peak displacement attenuation is depicted in Figure 4(b). As shown, the simulation results are in general agreement with the experimental data from several spherical wave experiments in granite.

The rebound phase in the velocity records is largely due to yielding and damage. As the main wave propagates outward from the source, the material behind the shock front first yields then fails due to the accumulation of damage. The damaged region encompasses a portion of the flow field nearest the charge cavity, while the yielded region extends further out into the flow field. Our simulations show that the material behavior during the rebound phase is strongly influenced by the impedance mismatch at the interface between the yielded and damaged regions: the larger the mismatch, the more prominent the rebound.

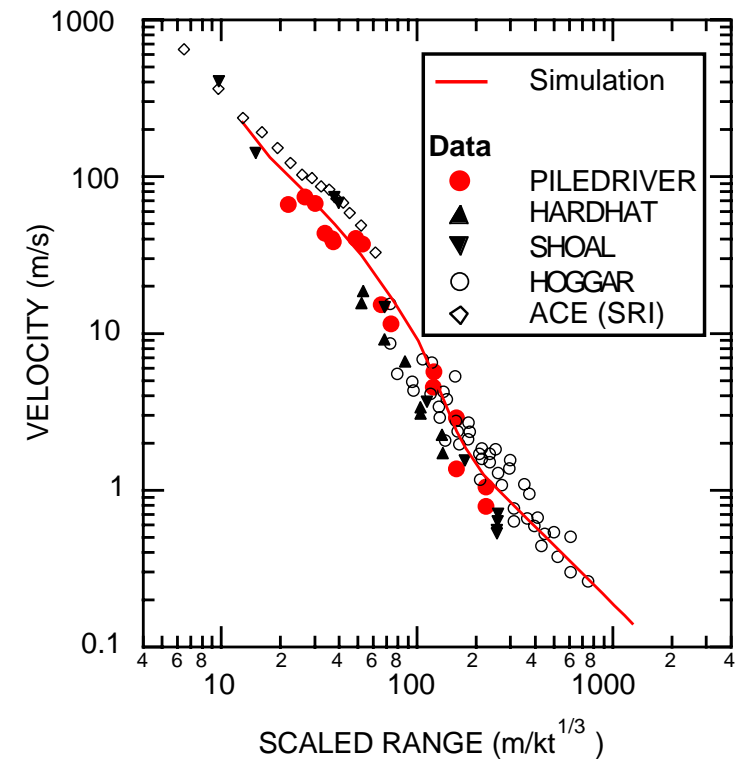

(a) Particle velocity

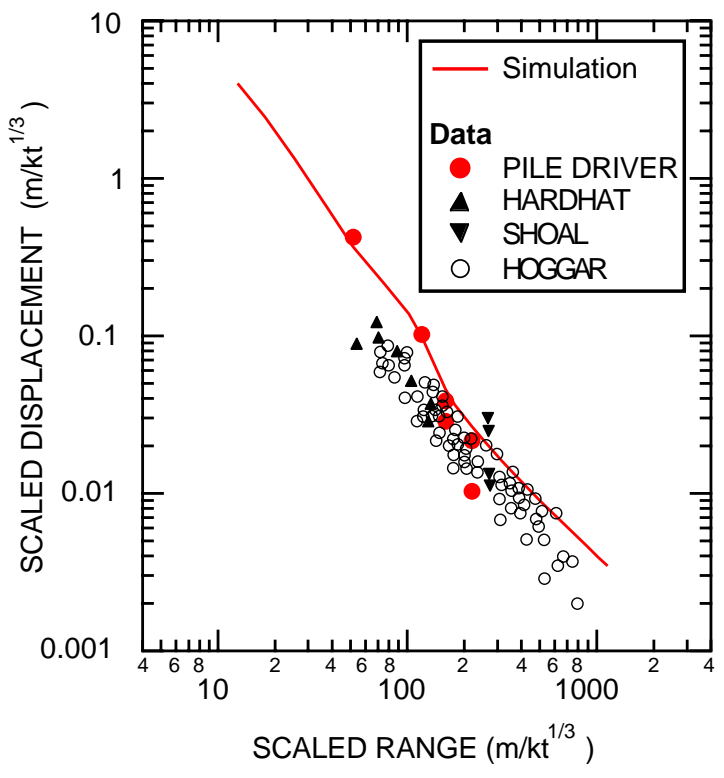

(b) Particle displacement

FIGURE 4. Comparison of simulated peak attenuation with measurements from several spherical wave experiments in granite.

\section{CONCLUSIONS}

A new scale-dependent strength and damage model has been developed which gives good agreement with both static tests and dynamic measurements of large scale motion caused by underground explosions. The model includes effects of bulking, pressure hardening and damage due to distortional deformations which are found to be important to simulate the material response especially in spherical loading. 


\section{ACKNOWLEDGMENTS}

Work performed under the auspices of the U. S. Department of Energy by the Lawrence Livermore National Laboratory under Contract W-7405-ENG-48.

\section{REFERENCES}

1. Rubin, M.B, Vorobiev, O.Yu., Glenn, L.A., accepted for publication in Int. J. Solids and Structures (1999)

2. Swegle, J. W. and Grady, D. E., J. Appl. Phys., 58,692-701 (1985)

3. Vorobiev, O. Yu, Glenn, L. A., Rubin, M. B., Simulation of the dynamic response of porous elastic-viscoplastic materials, to be published

4. Schock, R. N., Heard, H. C., and Stephens, D. R., J. Geophys. Res., 78(36), 5922-5941 (1973).

5. Stevens, J. L., Rimer, N., and Day, S. M., "Constraints on Modeling of Underground Explosions in Granite," Report SSS-R-87-8312, S-Cubed division, Maxwell Labs (1986).

6. $\quad$ Lamb, F.K., Callen,B.W., Sullivan,J.D., J.Geophys.Res., 97, 515-535 (1992)

7. Perret, W.R.,"Free Field Ground Motion in Granite'", Report No.POR-4001, Sandia Laboratory, Albuquerque, New Mexico (1968)

8. Vorobiev, O.Yu, Tarabay H.A, Lomov I.N, Glenn, L.A, "A Strength and damage model for rock under dynamic loading", to be published in Proc. APS Topical Conference on Shock Compression of Condensed Matter, Snowbird, Utah, June27-July2, 1999

9. Lockner D.A, "A generalized law for brittle deformation of Westerly granite", J.of Geophys.Res., 103(B3), 51075123 (1998) 